\title{
Mutismo acinético relacionado con hidrocefalia y cirugía cerebelosa tratado con bromocriptina y efedrina. Revisión fisiopatológica
}

\author{
O. Mateo-Sierra; F.A. Gutiérrez; C. Fernández-Carballal; D. Pinilla; B. Mosqueira; B. Iza y R. Carrillo
}

Servicio de Neurocirugía. Hospital General Universitario Gregorio Marañón. Madrid.

\section{Resumen}

El mutismo acinético (MA) es un trastorno del comportamiento, caracterizado por la incapacidad para moverse o hablar en pacientes despiertos. Típicamente ha sido descrito como una complicación transitoria de la cirugía de tumores de fosa posterior. Sin embargo, el MA también puede aparecer tras múltiples fallos valvulares en pacientes hidrocefálicos. En estos casos, el MA no mejora espontáneamente, ni con revisiones valvulares, pero puede responder al tratamiento con bromocriptina. Presentamos una paciente con MA tras una cirugía de fosa posterior, complicada por una ventriculitis con dilataciones ventriculares repetidas, que sólo mejoró con bromocriptina. Revisamos la fisiopatología del MA. Aunque ésta no sea bien conocida, parece que la afectación del núcleo dentado y de sus eferencias (principalmente de glutamato) sería responsable del MA de origen cerebeloso, mientras que la afectación de las vías monoaminérgicas paraventriculares explicaría el MA relacionado con dilataciones ventriculares repetidas y que responde al tratamiento con bromocriptina. Aun así, se requiere un estudio más profundo de esta patología para aclarar su etiología.

PALABRAS CLAVE: Mutismo acinético. Bromocriptina. Agonista dopaminérgico. Fosa posterior. Hidrocefalia.

Akinetic mutism related to hydrocephalus and cerebellar surgery treated with bromocriptine and ephedrine. A pathophysiological review

\section{Summary}

Akinetic mutism (AM) is a behavioral disorder characterized by impossibility to move or speak in awake patients. It has been typically described as a transient disorder following posterior fossa tumour resection. Besides, AM may also appear after recurrent shunt

Recibido: 03-06-04. Aceptado: 18-08-04 failures in hydrocephalic patients, with no tendency towards improvement, either spontaneously or with shunt revisions. However successful treatment of this second type of AM has been achieved with bromocriptine. We present a patient who developed AM after a posterior fossa surgery complicated by ventriculitis and multiple hydrocephalic events. AM only improved with bromocriptine. We review AM pathophysiology. Although not well known, it appears to be quite different, depending on its cerebellar or hydrocephalic origin. Damage to dentate nucleus or its efferents (mainly of glutamate) should promote AM of cerebellar origin, while damage to paraventricular monoaminergic pathways could explain AM related to repeated shunt failures which has successful response to bromocriptine treatment. However, a more complete study of this disorder is required to ascertain its aetiology.

KEY WORDS: Akinetic mutism. Bromocriptine. Dopamine agonist. Posterior fossa. Hydrocephalus

\section{Introducción}

El mutismo acinético (MA) es un trastorno poco frecuente de la motivación, en el cual el paciente se encuentra en estado de vigilia, pero no presenta actividad motora voluntaria ni del lenguaje, pudiendo considerarse el caso extremo del trastorno abúlico.

Este síndrome ha sido descrito en la literatura en casos de hidrocefalia con múltiples revisiones valvulares, sin relación con una malfunción valvular. En estos caso, los cambios de volumen ventricular repetitivos afectan a las vías monoaminérgicas ascendentes paraventriculares, pudiendo desencadenar el $\mathrm{MA}^{1,3,4,13,15,18,28}$. Este mecanismo fisiopatológico explicaría la buena respuesta obtenida en algunos caso con el tratamiento con análogos monoaminérgicos, que contrasta con la ausencia de mejoría con las

Abreviaturas. AM: akinetic mutism. MA: mutismo acinético. BC: bromocriptina. DUP: derivación ventriculoperitoneal. GCS: Glasgow Coma Scale. 

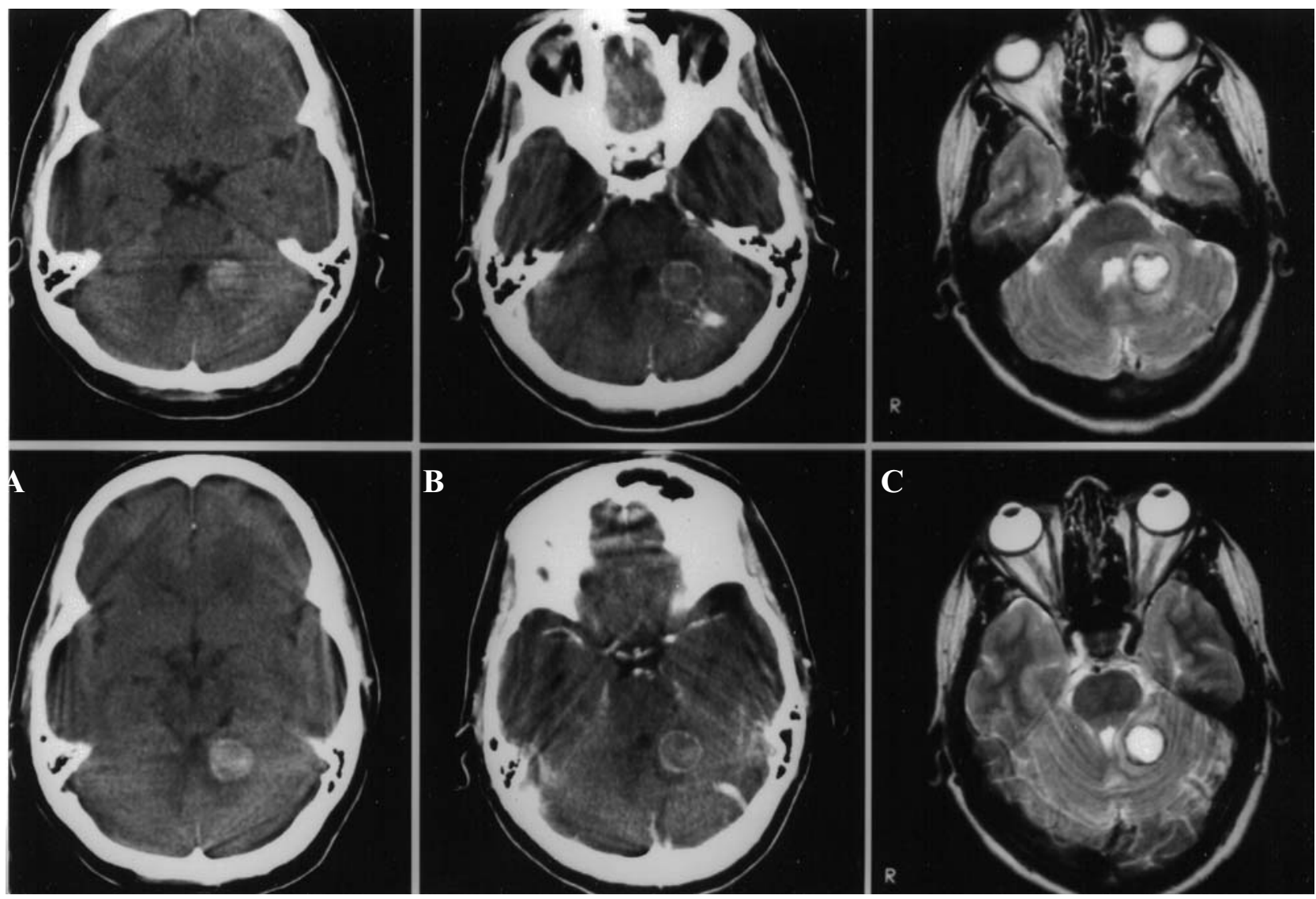

Figura 1. TC y RM craneales iniciales: Fig. 1a: TC craneal simple: lesión hemorrágica cerebelosa profunda en región paravermiana izquierda. Fig. l.b: TC craneal con contraste: captación del contraste en periferia de la lesión descrita y existencia de un angioma venoso cercano. Fig. l.c: RM craneal: lesión con signos de sangrado subagudo y crónico compatible con cavernoma cerebeloso.

revisiones valvulares ${ }^{19,24}$.

Por otra parte, el MA también puede aparecer como complicación de la cirugía de fosa posterior, pero con una fisiopatología que parece ser diferente $e^{5,8,10,11,23,26}$. En diferentes series se ha descrito que las lesiones del núcleo dentado o de sus conexiones (las cuales no son preferentemente monoaminérgicas ${ }^{9}$ ) podrían desencadenar el MA.

Presentamos una paciente con MA tras una cirugía de fosa posterior, complicada con hidrocefalias repetidas, que respondió al tratamiento con bromocriptina y efedrina. Se discuten las causas del mutismo en esta paciente y las diferentes posibilidades terapéuticas mediante una revisión de la literatura.

\section{Caso clínico}

Mujer de 45 años que ingresó por cefalea de inicio súbito, asociada a un síndrome cerebeloso (ataxia y dismetría izquierda). En los estudios de imagen, TC y RM craneal (Figura 1), se evidenció un cavernoma cerebeloso paravermiano izquierdo que había sangrado.

La cirugía se realizó mediante una craniectomía suboccipital paramedial izquierda con resección, con guía estereotáxica, del cavernoma. En el postoperatorio inicial, la exploración fue completamente normal. A los dos días de la cirugía apareció un deterioro de nivel de conciencia por infección profunda de la herida y meningitis por Staphylococcus aureus. Se realizó un TC craneal urgente en el que se identificaron resección del cavernoma y cambios postquirúrgicos sin dilatación ventricular (Figura 2). Se inició tratamiento antibiótico intensivo, a pesar del cual se produjo una intensa ventriculitis con situación neurológica de coma profundo (GCS3/15). En los TC craneales evolutivos se objetivaron signos de ventriculitis e hidrocefalia con tabicaciones (Fig. 3). Ambas respondieron a la inserción repetida de drenajes con lavado y antibióticos.

Al desaparecer la infección, la paciente presentaba una exploración característica de mutismo acinético, con ausencia de conexión con el medio y apertura ocasional de ojos. La hidrocefalia tetracameral persistió en la RM y se pro- 

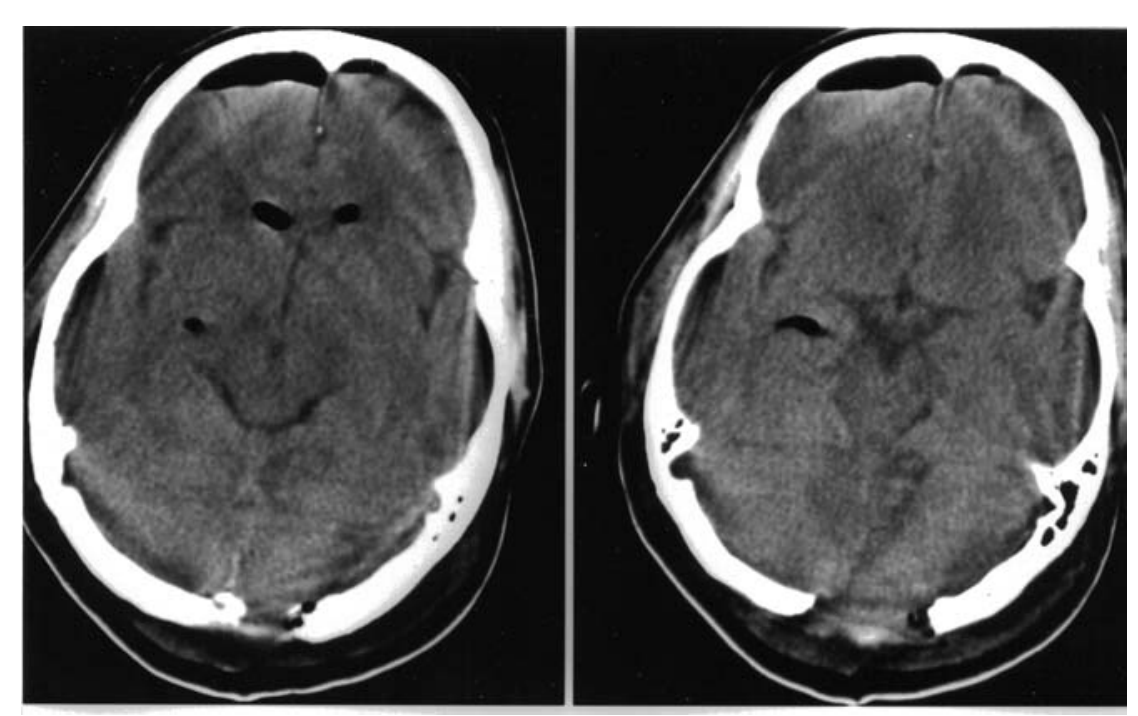

2005; 16: 134-141

Figura 2. TC craneal postquirúrgico tras inicio de infección profunda de la herida: craniectomía suboccipital paramediana izquierda con cambios postquirúrgicos en cerebelo. Resección del cavernoma, con cisternas basales presentes y discreto neumoencéfalo sin dilatación ventricular.
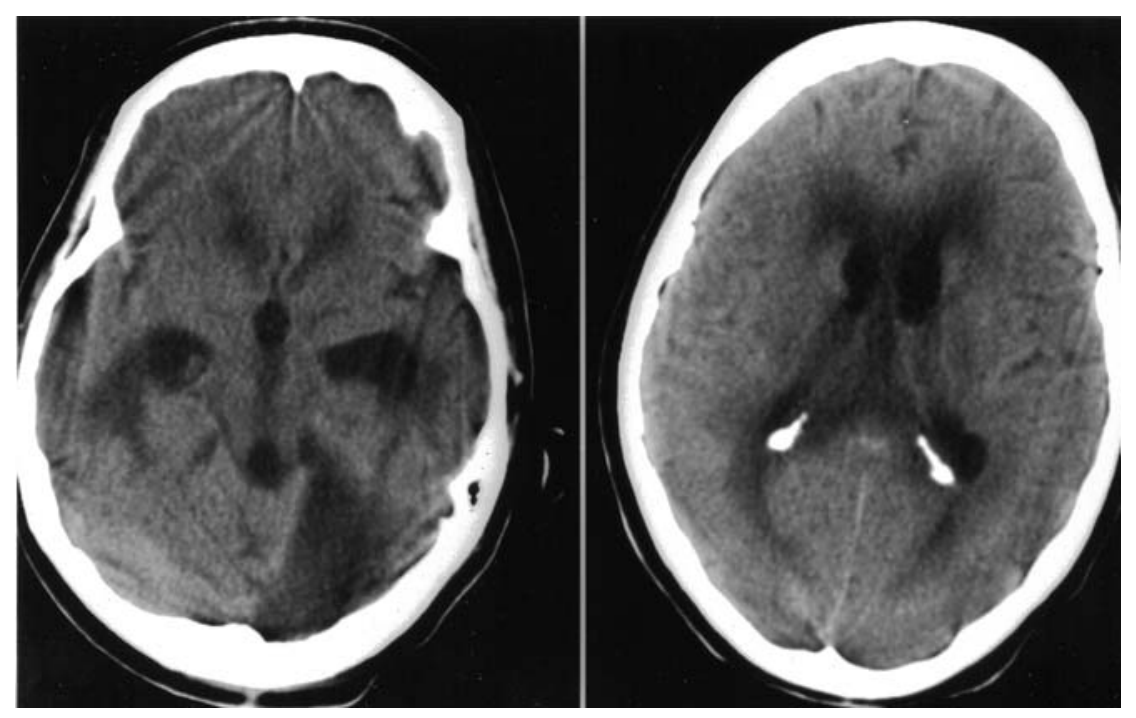

Figura 3. TC craneal tras ausencia de mejoría con tratamiento antibiótico: dilatación ventricular holocameral y de cisterna supracerebelosa izquierda con signos de ventriculitis difusa.
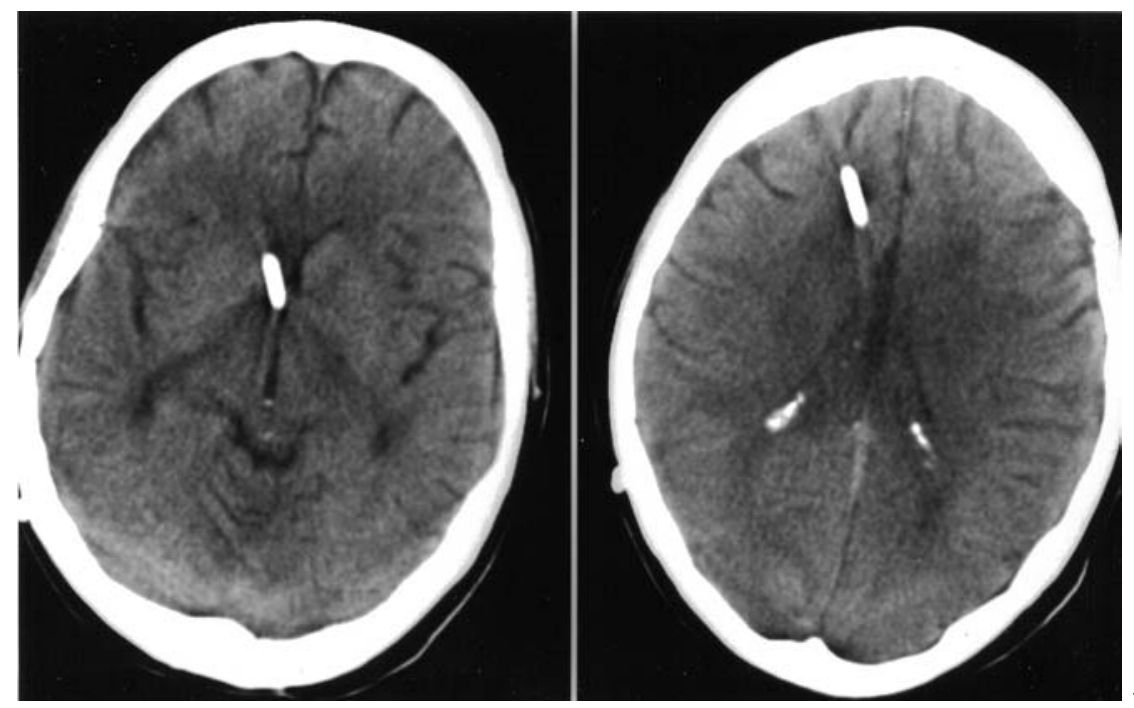

Figura 4. TC craneal a los dos días de la inserción de la DVP: ausencia de hidrocefalia y de signos activos de infección. 
cedió a la inserción de una derivación ventriculoperitoneal (DVP). A pesar de la corrección del tamaño ventricular con ésta, no se logró ninguna respuesta clínica tras 15 días de adecuado funcionamiento valvular (Figura 4).

Posteriormente se inició tratamiento con Bromocriptina (BC), con dosis iniciales de 2,5 mg cada 12 horas, duplicando la dosis cada dos días; cuando la dosis alcanzó los $20 \mathrm{mg} /$ día fue evidente una mejoría clínica con conexión periódica con el medio, emisión de monosílabos y apertura ocular frecuente; al subir a $40 \mathrm{mg}$ /día la paciente conectaba casi permanentemente con el medio, comenzaba a sonreír, decía frases cortas, movilizaba las cuatro extremidades, y controlaba la sedestación. Dada la ausencia de efectos secundarios se aumentó la dosis hasta los $100 \mathrm{mg}$ /día descritos por Anderson, llegándose a iniciar la alimentación y deambulación con ayuda, así como la emisión de frases complejas.

Para descartar el efecto placebo de la medicación o la evolución natural de su recuperación se procedió a disminuir las dosis de $\mathrm{BC}$, desencadenándose un deterioro clínico significativo que desapareció al reiniciar la dosis previa.

Tras alcanzar los $100 \mathrm{mg}$ diarios de $\mathrm{BC}$, se asoció la Efedrina, en dosis de $50 \mathrm{mg} / \mathrm{d}$. El estado neurológico con estas dosis era de plena conciencia, buena conexión con el medio, control de esfínteres, y deambulación más ágil. Sin embargo, la aparición de taquiarritmias graves obligó a retirar la Efedrina, con un discreto deterioro posterior.

A los 18 meses de seguimiento, la paciente precisa iguales dosis de medicación para mantener estable su condición neurológica. Sin embargo, sigue presentando aun importantes limitaciones cognitivas residuales.

\section{Discusión}

El mutismo acinético (MA) es una patología muy poco frecuente caracterizada por un trastorno fundamental del lenguaje con ausencia total de emisión del mismo, asociado a un estado abúlico de difícil tratamiento. Actualmente, se ha relacionado con diversas patologías neurológicas, como son la patología de fosa posterior-8, ${ }^{5-10-}$ $12,14,22,23,26,27$, las hidrocefalias repetidas ${ }^{1,3,4,13,15,18,28}$, y los trastornos isquémicos/tumorales bilaterales ${ }^{2,13,16,17,20,21,25}$, pero su fisiopatología y tratamiento son aún materia de debate.

El MA asociado a lesiones de fosa posterior se ha revisado en diferentes series, destacando las de Ildan y cols., y Gelabert y cols., de 117 y 157 pacientes, respectivamente. Aunque aparece con mayor frecuencia tras cirugía de tumores de línea media ${ }^{5,6,8,10,11,23,26}$, también se ha descrito en otros tipos de patología de fosa posterior no quirúrgica como cerebelitis y síndrome hemolítico-urémico ${ }^{14}$, enfermedades neurológicas ${ }^{7}$, estado post-traumático ${ }^{12}$, o tras hemorragia en tronco cerebral $^{27}$, con un mismo perfil clínico.

En general, se inicia en el postoperatorio inmediato ${ }^{23} \mathrm{o}$ en los primeros días ${ }^{5,6,10}$, con tendencia a la recuperación progresiva al cabo de varias semanas o meses (Tabla 1), aunque con persistencia de secuelas cerebelosas ${ }^{6,22,26}$. La recuperación espontánea, lo largo del tiempo, es la norma y es un hecho que diferencia este tipo de MA de otros.

Existe controversia acerca de cuál es el núcleo responsable de la aparición del MA. Tanto el núcleo dentado como los núcleos pontinos, el tálamo ventrolateral y el córtex motor, sensitivo y motor suplementario están implicados en la activación motora y del lenguaje. La mayoría de los autores considera que el MA sería secundario a una disfunción cerebelosa global con una mayor lesión del núcleo dentado ${ }^{14,22,23}$, o de las conexiones del núcleo dentado con estructuras pontinas, talámicas y corticales ${ }^{8,10-12,26}$. Sin embargo, también se ha descrito en lesiones aisladas del haz dentotalámico ${ }^{27} \mathrm{o}$ de las conexiones extracerebelo$\operatorname{sas}^{7,26}$

Dado que los principales neurotransmisores empleados en las proyecciones dento-talámicas corticales son el glutamato y el aspartato ${ }^{9}$, parece razonable pensar que en MA por lesiones puramente cerebelosas o de su vía eferente principal los análogos monoaminérgicos no serían útiles. Por el contrario, la respuesta significativa a bromocriptina en los casos de Caner y Echeverri, podría derivarse de la asociación de la patología cerebelosa a dilatación ventricular o lesión del tronco cerebral.

En la paciente aquí presentada, el antecedente de cirugía de fosa posterior paramedial podría ser el origen del MA. Sin embargo, no reúne las características típicas. Aparece en el $2^{\circ}$ día de postoperatorio, pero en el contexto de un deterioro global neurológico, no cerebeloso. No mejora espontáneamente y precisa dosis muy altas y mantenidas de bromocriptina (100 mg/día), en comparación con el paciente de Caner $(7,5 \mathrm{mg} / \mathrm{d})$. Por ello nos planteamos otro mecanismo causal en esta paciente, tomando en consideración las dilataciones ventriculares repetidas detectadas.

El MA ha sido diagnosticado en pacientes con episodios repetidos de hidrocefalia (Tabla 2) y se considera secundario a la lesión de las vías monoaminérgicas paraventriculares, especialmente dopaminérgicas $\mathrm{y}$, en menor medida, noradrenérgicas ${ }^{1,3,4,13,15,18,28}$. De hecho, este mecanismo se ha reproducido con éxito en animales de experimentación y se ha comprobado su respuesta favorable a agonistas dopaminérgicos. Este estudio sirvió de base para su introducción en la clínica por Ross y Stewart ${ }^{19,24}$.

Aunque en algunas ocasiones, el mutismo puede revertirse inicialmente con la revisión de la derivación ${ }^{4}$, en general este tipo de MA no mejora, a pesar de la normalización de los volúmenes ventriculares, y sí responde a medidas farmacológicas ${ }^{1,3,4,13,15,18,28}$.

El empleo de bromocriptina (BC) a dosis altas ha 


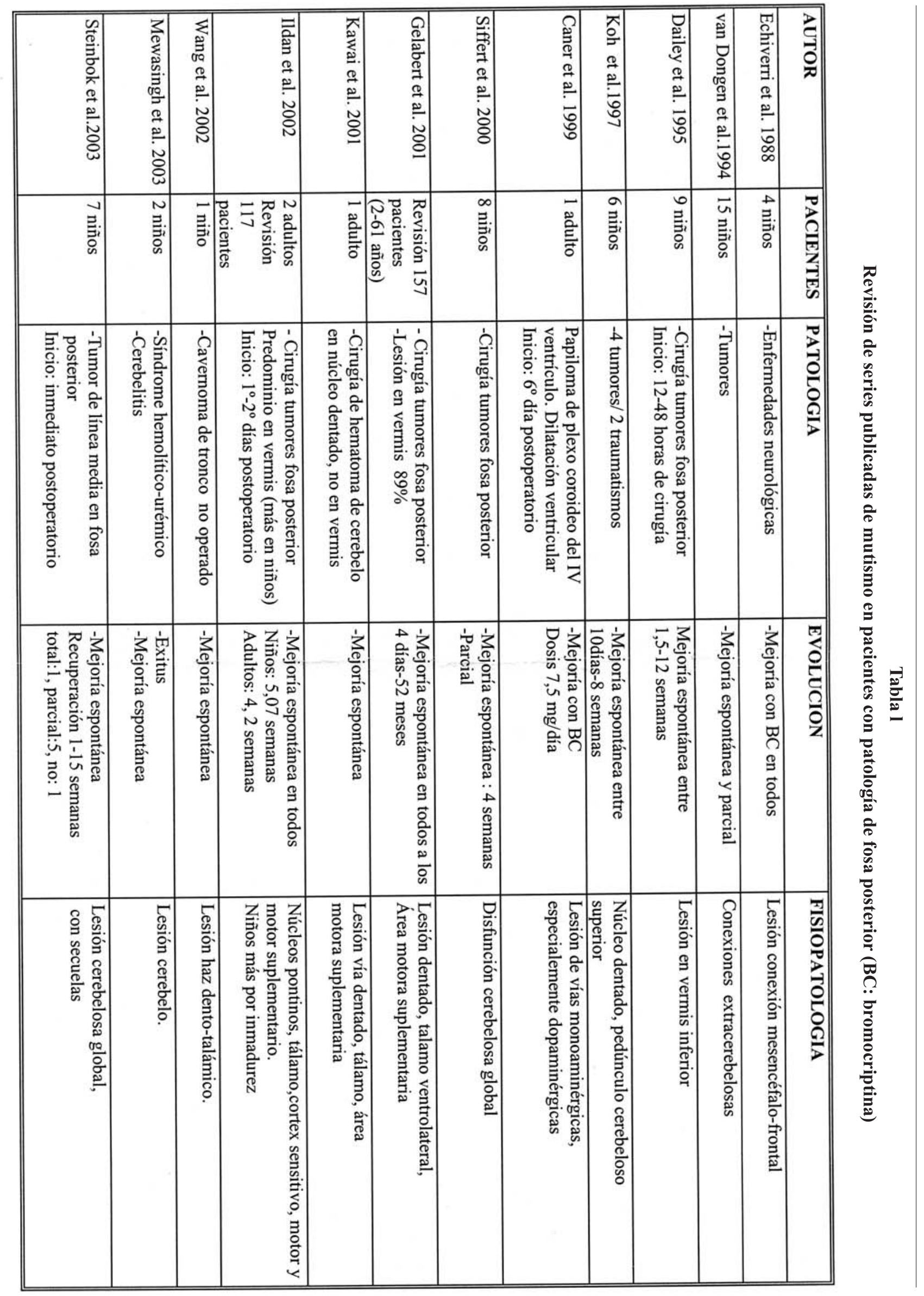




\section{Tabla 2}

Revisión de series publicadas de mutismo acinético relacionado con hidrocefalia (PSP: parálisis supranuclear progresiva; BC: bromocriptina).

\begin{tabular}{||l|l|l||}
\hline AUTOR & PATOLOGIA & EVOLUCION \\
\hline $\begin{array}{l}\text { Berger et al. } \\
1985\end{array}$ & $\begin{array}{l}\text { 1 paciente } \\
\text { Hidrocefalia repetida }\end{array}$ & $\begin{array}{l}\text { Asocia parkinsonismo } \\
\text { Mejora con DA }\end{array}$ \\
\hline $\begin{array}{l}\text { Watahiki et al. } \\
1987\end{array}$ & $\begin{array}{l}\text { 1 paciente } \\
\text { Hidrocefalia repetida }\end{array}$ & $\begin{array}{l}\text { Mejoría con BC, L-DOPA } \\
\text { yrihexifenidilo }\end{array}$ \\
\hline $\begin{array}{l}\text { Anderson et al. } \\
1992\end{array}$ & $\begin{array}{l}\text { 1 paciente } \\
\text { Hidrocefalia repetida }\end{array}$ & $\begin{array}{l}\text { Mejoría con BC y } \\
\text { Efedrina }\end{array}$ \\
\hline $\begin{array}{l}\text { Moser et al. } \\
1995\end{array}$ & $\begin{array}{l}\text { 1 paciente } \\
\text { Hidrocefalia repetida }\end{array}$ & $\begin{array}{l}\text { Mejoría con BC y } \\
\text { Metoprolol }\end{array}$ \\
\hline $\begin{array}{l}\text { Lin et al. } \\
1997\end{array}$ & $\begin{array}{l}\text { 2 pacientes } \\
\text { Hidrocefalia repetida } \\
\text { Cavum septum pellucidum }\end{array}$ & $\begin{array}{l}\text { 1 mejora } \\
\text { no responde a BC } \\
\text { (persiste cavum??) }\end{array}$ \\
\hline $\begin{array}{l}\text { Aidi et al. } \\
2000\end{array}$ & $\begin{array}{l}\text { 2 pacientes } \\
\text { Hidrocefalia repetida } \\
2003\end{array}$ & $\begin{array}{l}\text { Asocia PSP } \\
\text { Mejora con BC }\end{array}$ \\
\hline
\end{tabular}

Tabla 3

Revisión de series publicadas de mutismo por otras causas (excluyendo hidrocefalias repetidas o patología cerebelosa)

\begin{tabular}{|c|c|}
\hline AUTOR & CAUSA \\
\hline Nemeth et al. 1988 & 3 pacientes: Lesión cingular anterior bilateral \\
\hline Shinoda et al. 1993 & $\begin{array}{l}1 \text { paciente: Lesión post-traumática en tálamo } \\
\text { izquierdo e hipotálamo derecho }\end{array}$ \\
\hline Ure et al. 1998 & $\begin{array}{l}1 \text { paciente: Necrosis bilateral del pálido } \\
1 \text { paciente: Necrosis de pálido izquierdo e infarto fronto- } \\
\text { parietal derecho } \\
1 \text { paciente: Infarto estriato-capsular }\end{array}$ \\
\hline Rubin et al. 1999 & 1 paciente: Toxicidad por baclofén \\
\hline Alexander 2001 & 1 paciente: Infarto mesencéfalo-diencefálico bilateral \\
\hline Oberndofer et al.2002 & 1 paciente: Infiltración tumoral bilateral del fornix. \\
\hline
\end{tabular}


demostrado su eficacia con resolución del MA en los escasos casos publicados ${ }^{1,13,18}$. Otros medicamentos que han resultado útiles en esta patología son la dopamina ${ }^{4}$, o la combinación de $\mathrm{BC}$ con efedrina ${ }^{3}$, con metoprolol ${ }^{15}$, y con L-dopa y trihexifenidilo ${ }^{28}$. Sin embargo, la posibilidad de efectos secundarios derivados del uso de alguno de estos fármacos limita su utilidad.

El MA, en nuestro caso, parece relacionarse más estrechamente con la patología derivada de la ventriculitis e hidrocefalias repetidas que con la cirugía de fosa posterior. La respuesta clínica obtenida con dosis progresivamente mayores de BC avalan esta teoría. La dependencia en cantidad y a lo largo del tiempo de dichas dosis, como en el caso publicado por Anderson, hace más probable esta hipótesis fisiopatológica.

\section{Bibliografía}

1. Aidi, S., Elalaoui-Faris, B., Benomar, A., Chaoui, M., Chkili, T.: Akinetic mutism and progressive supranuclear palsy-like syndrome after the shunt of an obstructive hydrocephalus. Successful treatment with bromocriptine: 2 cases. Rev. Neurol. (Paris) 2000; 156: 380-383.

2. Alexander, M.P.: Chronic akinetic mutism after mesencephalic-diencephalic infarction: remediated with dopaminergic mediactions. Neurorehabil. Neural Repair 2001; 15 : 151-156.

3. Anderson, B.: Relief of akinetic mutism from obstructive hydrocephalus using bromocriptine and ephedrine. J. Neurosurg. 1992; 76: 152-155.

4. Berger, L., Gauthier, S., Leblanc, R.: Akinetic mutism and parkinsonism associated with obstructive hydrocephalus. Can. J. Neurol. Sci. 1985; 12: 255-258.

5. Caner, H., Altinors, N., Benli, S., Calisaneller, T., Albayrak. A.: Akinetic mutism after fourth ventricle choroid plexus papilloma: treatment with a dopamine agonist. Surg. Neurol. 1999. 51: 181-184.

6. Dailey, A.T., McKhann, G.M.2 ${ }^{\text {nd }}$., Berger, M.S.: The pathophysiology of oral pharyngeal apraxia and mutism following posterior fossa tumor resection in children. J. Neurosurg. 1995; 83: 467-475.

7. Echiverri, H.C., Tatum, W.O., Merens, T.A., Coker, S.B.: Akinetic mutism: pharmacologic probe of the dopaminergic mesencephalofrontal activating system. Pediatr. Neurol. 1988; 4: 228-230.

8. Gelabert-Gonzalez, M., Fernandez-Villa, J.: Mutism after posterior fossa surgery. Review of the literature. Clin. Neurol. Neurosurg. 2001; 103: 111-114.

9. Haines, D.E., Mibailoff, G.A., Bloedel, J.R.: Cerebelo. En D.E.Haines (ed). Principios de Neurociencia. Madrid; Elsevier Science, 2003; 423-444.

10. Ildan, F., Tuna, M., Erman, T., Gocer, A.I., Zeren, M., Cetinalp, E.: The evaluation and comparison of cerebellar mutism in children and adults alter posterior fossa surgery: report of two adult cases and review of the literature. Acta Neurochir. (Wien.) 2002; 144: 463-473.

11. Kawai, H., Ohta, F., Matsumoto, Y., Yamamoto, Y.: An adult case of cerebellar mutism alter renoval of cerebellar hematoma. No to Shinkei 2001; 53: 475-479.

12. Koh, S., Turkel, S.B., Baram, T.Z.: Cerebellar mutism in children: report of six cases and review of the literature. Pediatr. Neurol. 1997; 16; 218-219.

13. Lin, K.L., Wang, H.S., Chou, M.L., Rui, T.N.: Role of cavum septum pellucidum in akinetic mutism of hydrocephalic children. Pediatr. Neurol. 1997; 16: 156-159.

14. Mewasingh, L.D:, Kadhim, H., Christophe, C., Chistiaens, F.J., Dam, B.: Nonsurgical cerebellar mutism (anarthria) in two children. Pediatr. Neurol. 2003; 28: 59-63.

15. Moser, A., Freyberger, H.J., Bruckmann, H., Kompf, D.: Akinetic mutism in decompensated triventricular hydrocephalus. Fortschr. Neurol. Psychiatr. 1995 ; 63 : 248-251.

16. Nemeth, G., Hegedus, K., Molnar, L.: Akinetic mutism associated with bicingular lesions: clinicopathological and functional anatomical correlates. Eur. Arch. Psychiatry Neurol. Sci. 1998; 237: 218-222.

17. Oberndofer, S., Urbanits, S., Lahrmann, H., Kirschner, H., Kumpan, W., Grisold, W.: Akinetic mutism caused by a bilateral infiltration of the fornix in a patient with astrocytoma. Eur. J. Neurol. 2002 ; 9: 311-313.

18. Psarros, T., Zouros, A., Coimbra, C.: Bromocriptine-responsive akinetic mutism following endoscopy for ventricular neurocysticercosis. Case report and review of the literature. J. Neurosurg. 2003; 99: 397-401.

19. Ross, E.D., Stewart, R.M.: Akinetic mutism from hypothalamic damage: successful treatment with dopamine agonists. Neurology 1981; 31: 1435-1439.

20. Rubin, D.L., So, E.L.: Reversible akinetic mutism possibly induced by baclofen. Pharmacotherapy. 1999; 19: 468-470.

21. Shinoda, M., Tsugu, A., Oda, S. et al.: Development of akinetic mutism and hyperphagia alter left thalamic and right hypothalamic lesions. Childs Nerv. Syst. 1993; 9: 243-245.

22. Siffert, J., Poussaint, T.Y., Goumnerova, L.C., et al.: Neurological dysfunction associated with postoperative cerebellar mutism. J. Neurooncol. 2000; 48: 75-81

23. Steinbok, P., Cochrane, D.D., Perrin, R., Price, A.: Mutism after posterior fossa tumour resection in children: incomplete recovery on long-term follow-up. Pediatr. Neurosurg. 2003; 39: 179-183.

24. Stewart, J.T., Leadon, M., González-Rothi, L.J.: Treatment of a case of akinetic mutism with bromocriptine. J. Neuropsychiatry. Clin. Neurosci. $1990 ; 2$ :462-463.

25. Ure, J., Faccio, E., Videla, H. et al.: Akinetic mutism: a report of three cases. Acta Neurol. Seand. 1998; 98: 439-444.

26. Van Dongen, H.R., Catsman-Berrevoets, C.E., van Mourik, M.: The syndrome of cerebellar mutism and subse- 
quent dysarthria. Neurology 1994; 44: 2040-2046.

27. Wang, M.C., Winston, K.R., Breeze, R.E.: Cerebellar mutism associated with a mid-brain cavernous malformation. Case report and review of the literature. J. Neurosurg. 2002; 96: 607-610.

28. Watahiki, Y., Narita, S., Kurahasi, K., Tanaka, T., Matsunaga, M.: Akinetic mutism from recurrent hydrocephalus: successful treatment with levodopa, bromocriptine and triheypheniddyl. No To Shinkei 1987; 39: 977-982.

\section{Comentario al trabajo: Mutismo acinético relacionado con hidrocefalia y cirugía cerebelosa tratado con bromo- criptina y efedrina. Revisión fisiopatológica de Mateo- Sierra y cols.}

Mateo y cols. presentan el caso de una paciente adulta sometida a intervención quirúrgica sobre el hemisferio cerebeloso izquierdo por un cavernoma que al segundo día de postoperatorio inicia sintomatología de meningitis-ventriculitis con depresión del nivel de consciencia hasta GCS 3/15, Las TC craneales evolutivas mostraron dilatación ventricular. El cuadro clínico se resolvió tras tratamiento antibiótico e inserción de drenajes ventriculares; sin embargo la paciente permaneció en situación de mutismo acinético que respondió al tratamiento con bromocriptina y efedrina.

Los autores realizan una revisión de la literatura exponiendo las principales situaciones relacionadas con la presentación de este cuadro clínico. Resulta de especial interés la diferenciación entre dos entidades de presentación clínica similar pero con base fisiopatológica, y por ello terapéutica, probablemente distintas. En primer lugar, el llamado mutismo cerebeloso, que acontece tras cirugía de lesiones de fosa posterior, fundamentalmente vermianas o paravermianas, de tamaño considerable, y más frecuentemente descrito en pacientes en edad pediátrica, quizás por la alta prevalencia de lesiones tumorales de esta localización. En estos casos, la causa parece ser la lesión de las aferencias y eferencias del núcleo dentado, así como fenómenos de diasquisis (alteración cruzada del flujo sanguíneo). La mayoría de estos cuadros mejoran espontáneamente en semanas, aunque con persistencia de una disartria grave en la mayoría de los pacientes ${ }^{2,3}$. En segundo lugar, el mutismo acinético relacionado con dilataciones ventriculares de repetición en casos de hidrocefalias complejas, que ha sido atribuido a lesión mesencefálica y de las vías monoaminérgicas paraventriculares. En estos casos,
Mateo-Sierra, O.; Gutiérrez, F.A.; Fernández-Carballal, C.; Pinilla, D.; Mosqueira, B.; Iza, B.; Carrillo, F.: Mutismo acinético relacionado con hidrocefalia y cirugía cerebelosa tratado con bromocriptina y efedrina. Revisión fisiopatológica. Neurocirugía 2005; 16: 134-141.

Correspondencia postal: Olga Mateo-Sierra. Servicio de Neurocirugía. Hospital General Universitario "Gregorio Marañón". C/ Dr. Esquerdo 46. 28007-Madrid los pacientes responden favorablemente al tratamiento con bromocriptina, dopamina, efedrina, etc. ${ }^{1}$.

El caso que presentan los autores es difícilmente encuadrable en una de las dos entidad, puesto que el cuadro clínico aparece a las 48 horas de la intervención quirúrgica de una lesión cerebelosa izquierda en el contexto de un proceso infeccioso (meningitis y ventriculitis), $\mathrm{y}$ antes de poderse evidenciar la presencia de dilataciones ventriculares en la TC. Es probable por ello, que el dramático deterioro del nivel de consciencia de la paciente sea, inicialmente, atribuible al proceso séptico. Sin embargo, una vez resuelta la infección, la paciente permanece en situación de mutismo acinético que responde muy favorablemente a dosis crecientes de bromocriptina, lo que apuntaría hacia un mutismo por afectación de las vías monoaminérgicas paraventriculares como hipótesis fisiopatológica más probable.

\section{Bibliografía}

1. Abekura, M.: Akinetic mutism and magnetic resonance imaging in obstructive hydrocephalus. Case illustration. Neurosurgery 1998; 88: 161.

2. Riva, D., Giorgi, C.: The cerebellum contributes to higher functions during development. Brain 2000; 123: 10511061

3. Sagiuchi, T., lshii, K., Auki, Y., et al.: Bilateral crossed cerebello-cerebral diaschisis and mutism after surgery for cerebellar medulloblastoma. Ann Nucl Med. 2001; 15: 157160 .

M. Brell

Barcelona 